\title{
A precise and accurate determination of the cosmic microwave background temperature at $\mathbf{z}=\mathbf{0 . 8 9}$
}

\author{
S. Muller ${ }^{1}$, A. Beelen ${ }^{2}$, J. H. Black ${ }^{1}$, S. J. Curran ${ }^{3,4}$, C. Horellou ${ }^{1}$, S. Aalto ${ }^{1}$, F. Combes ${ }^{5}$, \\ M. Guélin ${ }^{6,7}$, and C. Henkel ${ }^{8,9}$
}

\author{
1 Department of Earth and Space Sciences, Chalmers University of Technology, Onsala Space Observatory, 43992 Onsala, Sweden \\ e-mail: mullers@chalmers.se \\ 2 Institut d'Astrophysique Spatiale, Bât. 121, Université Paris-Sud, 91405 Orsay Cedex, France \\ 3 Sydney Institute for Astronomy, School of Physics, The University of Sydney, 2006 NSW, Australia \\ ${ }^{4}$ ARC Centre of Excellence for All-sky Astrophysics (CAASTRO), Australia \\ 5 Observatoire de Paris, LERMA, CNRS, 61 Av. de l'Observatoire, 75014 Paris, France \\ ${ }^{6}$ Institut de Radioastronomie Millimétrique, 300 rue de la piscine, 38406 Saint-Martin d'Hères, France \\ 7 École Normale Supérieure/LERMA, 24 rue Lhomond, 75005 Paris, France \\ 8 Max-Planck-Institut für Radioastonomie, Auf dem Hügel 69, 53121 Bonn, Germany \\ 9 Astron. Dept., King Abdulaziz University, PO Box 80203, Jeddah, Saudi Arabia
}

Received 22 October 2012 / Accepted 21 December 2012

\begin{abstract}
Context. According to the Big Bang theory and as a consequence of adiabatic expansion of the Universe, the temperature of the cosmic microwave background (CMB) increases linearly with redshift. This relation is, however, poorly explored, and detection of any deviation would directly lead to (astro-)physics beyond the standard model.

Aims. We aim to measure the temperature of the CMB with an accuracy of a few percent at $z=0.89$ toward the molecular absorber in the galaxy lensing the quasar PKS 1830-211.

Methods. We adopted a Monte-Carlo Markov chain approach, coupled with predictions from the non-LTE radiative transfer code RADEX, to solve the excitation conditions of a set of various molecular species directly from their spectra.

Results. We determine $T_{\mathrm{CMB}}=5.08 \pm 0.10 \mathrm{~K}$ at $68 \%$ confidence level. Our measurement is consistent with the value $T_{\mathrm{CMB}}=5.14 \mathrm{~K}$ predicted by the standard cosmological model with adiabatic expansion of the Universe. This is the most precise determination of $T_{\mathrm{CMB}}$ at $z>0$ to date.
\end{abstract}

Key words. cosmology: observations - cosmic background radiation - cosmological parameters - quasars: absorption lines quasars: individual: PKS 1830-211

\section{Introduction}

\subsection{The CMB temperature as a function of redshift}

The cosmic microwave background (CMB) is one of the pillars of the Big Bang theory. This radiation field is a relic of the epoch when matter and photons decoupled at $z \sim 1100$ and fills the entire sky with a photon density of $\sim 400 \mathrm{~cm}^{-3}$. Since decoupling, the CMB photons have cooled down with the expansion of the Universe. Observations with the COBE satellite have demonstrated that the CMB corresponds to a nearly perfect black body, characterized by a temperature $T_{0}$ at $z=0$, which is measured with very high accuracy, $T_{0}=2.72548 \pm 0.00057 \mathrm{~K}$ (Fixsen 2009).

Measuring the CMB temperature at high redshift has considerable cosmological interests, in 1) demonstrating that the CMB radiation is universal (Equivalence principle) and 2) tracing the evolution of its temperature with redshift, $T_{\mathrm{CMB}}(z)$. Adiabatic expansion predicts that the $\mathrm{CMB}$ temperature evolution is proportional to $(1+z)$. Alternative cosmologies, such as decaying dark energy models (e.g. Lima 1996; Lima et al. 2000; Jetzer \& Tortora 2011), where dark energy can interact with matter via creation of photons, affecting the CMB spectrum, predict a deviation from this simple law with $T(z)=T_{0} \times(1+z)^{(1-\alpha)}$. Precise measurements of $T_{\mathrm{CMB}}$ at high redshift can therefore constrain such theories.

Two methods can be used to probe the CMB temperature at $z>0$. One is based on multi-frequency Sunyaev-Zeldovich (S-Z) observations toward galaxy clusters (see e.g. Horellou et al. 2005). Inverse Compton scattering by free electrons in a cluster of galaxies induces a distortion of the CMB spectrum, which can be used to constrain the CMB temperature at the location of the cluster. Battistelli et al. (2002) reported the first determination of $T_{\mathrm{CMB}}(z)$ toward the Coma $(z=0.02)$ and Abell 2163 $(z=0.2)$ clusters. More recently, this method was applied to a sample of 13 clusters between $z=0.023$ and $z=0.55$ by Luzzi et al. (2009), who found $\alpha=0.024_{-0.024}^{+0.068}$, thus a $T_{\mathrm{CMB}}-z$ law in agreement with the standard model. One limitation of the $\mathrm{S}-\mathrm{Z}$ method is the scarcity of clusters at high-redshift $(z>1)$. de Martino et al. (2012) discuss prospects in the near future based on Planck data.

The second method relies on spectroscopic studies of lines in absorption against quasars and their excitation analysis. Most of the measurements at high redshift have used UV spectroscopy of atomic species (C I: Meyer et al. 1986; Songaila et al. 1994b; Ge et al. 1997; Roth \& Bauer 1999; C II: Songaila et al. 1994a; Lu et al. 1996; Molaro et al. 2002; C I \& C II: Srianand et al. 2000). Strictly speaking, these measurements are all upper limits 
on $T_{\mathrm{CMB}}$, since the contributions from other local sources of excitation (collisions, local radiation field) are yet largely uncertain and poorly constrained, and so not fully counted for. More recently, Noterdaeme et al. (2011) observed electronic transitions of CO absorption toward several quasars at redshift between 1.7 and 2.7. Together with the previous measurements of $T_{\mathrm{CMB}}(z)$ in the literature, they could obtain the tightest constraint to date on the temperature evolution of the CMB, $\alpha=-0.007 \pm 0.027$, consistent with adiabatic expansion of the Universe. Nevertheless, all the studies so far have relied on a limited number of transitions/species, introducing a possible bias in the determination of excitation conditions. Also, the UV spectroscopy technique (e.g. of $\mathrm{CI}$ and $\mathrm{C}$ II) is not suitable below $z \sim 1$, due to the atmospheric cut-off at $300 \mathrm{~nm}$.

The use of radio-mm molecular absorbers to determine $T_{\mathrm{CMB}}$ at $z>0$ is another particularly attractive method, due to the tighter constraints on physical conditions and local excitation when a variety of molecular species is detected. In this paper, we follow this approach, with the aim of measuring the CMB temperature from multi-transition multi-species excitation analysis in the $z=0.89$ radio-mm molecular-rich absorber toward the quasar PKS 1830-211.

\subsection{The $z=0.89$ molecular-rich absorber toward PKS 1830-211}

PKS 1830-211 is a radio-loud blazar at a redshift $z=2.5$ (Lidman et al. 1999), gravitationally lensed by a $z=0.88582$ almost face-on spiral galaxy (Wiklind \& Combes 1996; Winn et al. 2002). The presence of an additional galaxy at $z=0.19$ along the line of sight is inferred from H I absorption (Lovell et al. 1996). The lensed image of the quasar appears in the radio continuum as a textbook example of a complete Einstein ring of $1^{\prime \prime}$ in diameter, plus two bright and compact components, located to the NE and SW of the ring (Jauncey et al. 1991). The Einstein ring has a steep spectral index and grows fainter with frequency, until invisible in the millimeter band. The NE and SW images of the quasar are marginally resolved in $43 \mathrm{GHz}$ VLBI observations with 0.5 mas beam resolution by Jin et al. (2003). At the distance of the $z=0.89$ galaxy, their angular size corresponds to a scale of $\sim 1 \mathrm{pc}$ at $\mathrm{mm}$ wavelengths. Most interestingly, the line of sight toward both images intercepts molecular clouds located in the spiral arms of the intervening $z=0.89$ galaxy, which results in remarkable absorption by a variety of molecular species (Wiklind \& Combes 1998; Menten et al. 1999; Muller et al. 2006). In fact, PKS 1830-211 is the strongest of only four redshifted mm-molecular absorbers known to date (Combes 2008), in addition to being the highest redshift and the most moleculerich (Muller et al. 2011). As such, PKS 1830-211 is a target of choice in using molecules as cosmological probes, for example of the CMB temperature, of the constancy of fundamental constants, or of the chemical evolution of the Universe (see e.g. Henkel et al. 2009; Muller 2011).

The activity of the blazar leads to time variations of its flux, with a time delay between the NE and SW images of about 25 days (Lovell et al. 1998; Wiklind \& Combes 1999), as well as changes in the morphology and barycenter of the continuum emission, seen in VLBI observations (Garrett et al. 1997; Jin et al. 2003). Nair et al. (2005) interpret the changes in the apparent distance between the NE and SW images by the recurrent ejection of plasmons along a helical jet in the blazar. Consequently, and of great importance for our study, the continuum emission of the two images is drifting behind the absorbing gas, at the rhythm of plasmon ejections. Jin et al. (2003) measured drifts up to $200 \mu$ as $(1.6 \mathrm{pc}$ in the lens plane at $z=0.89$ ) over eight months. The changes of sightline result in a time variation of the molecular absorption profiles, as revealed by the long-term monitoring of the $\mathrm{HCO}^{+} / \mathrm{HCN} 1-0$ lines by Muller \& Guélin (2008). This time variation could hamper studies which rely on the comparison of different molecular transitions, such as constraints on the constancy of fundamental constants, multi-transition excitation analysis, as well as abundance and ratio measurements.

\section{Observations and data reduction}

Our main goal was to observe several rotational transitions of various molecular species in order to derive their excitation and constrain the CMB temperature toward PKS 1830-211. In particular, to take advantage of the two SW/NE lines of sight through the intervening galaxy, which are independent (on either side of the bulge, at galactocentric distances of $\sim 2 \mathrm{kpc}$ and $\sim 4 \mathrm{kpc}$, respectively) and likely to have different excitation conditions, giving a robust measurement of $T_{\mathrm{CMB}}$ at $z=0.89$.

Toward the NE line of sight, only a handful of species have been detected (Muller et al. 2011): we have chosen to focus on the $\mathrm{HCO}^{+}, \mathrm{HCN}$, and $\mathrm{HNC} J=1-0,2-1$, and 3-2 transitions, all of which are optically thin. These same transitions are saturated or optically thick toward the SW line of sight, but their isotopic variants, observable in the same tunings, can be used instead. Several other species, such as $\mathrm{HOC}^{+}$and $\mathrm{SiO}$, also have rotational transitions in the same observable bands.

In order to cover the different rotational transitions of our targeted molecular species, we used the Australia Telescope Compact Array (ATCA) at $7 \mathrm{~mm}$ and $3 \mathrm{~mm}$ and the Plateau de Bure interferometer (PdBI) at $3 \mathrm{~mm}$ and $2 \mathrm{~mm}$. Details on the respective observations and specific data calibration are given hereafter in Sects. 2.1 and 2.2, and a summary of the observations is given in Table 1. Unfortunately, because of scheduling and weather constraints the ATCA and PdBI observations could not be carried out simultaneously, which raises the concern of time variations of the source between the observations. This problem is discussed in Sect. 2.3.

The full final spectra are shown in Figs. A.1 and A.2. A crude comparison of the new data and ATCA spectra obtained in 2009/2010 shows that significant variations have occurred (Fig. A.2): while the intensities of the SW absorption lines $\left(v=0 \mathrm{~km} \mathrm{~s}^{-1}\right)$ have increased by a factor of $\sim 2$ for most species, the absorption depths of the $\mathrm{HCO}^{+}$and $\mathrm{HCN}$ lines toward the NE image $\left(v=-147 \mathrm{~km} \mathrm{~s}^{-11}\right)$ have significantly decreased. The resulting low signal-to-noise ratio on these lines and the continuum baseline uncertainty due to the wings of the corresponding saturated lines from the SW component (see Fig. A.2), prevent us from obtaining a robust fit, which hampers our initial goal to derive $T_{\mathrm{CMB}}$ in several independent lines of sight.

Other velocity components (e.g. at velocity $-224 \mathrm{~km} \mathrm{~s}^{-1}$ and $+170 \mathrm{~km} \mathrm{~s}^{-1}$, see Muller et al. 2011), are also too weak to permit a meaningful analysis.

\subsection{Australia Telescope Compact Array (ATCA)}

Observations were carried out with the ATCA on 2011 July 27, with four antennas in a compact configuration. The longest baseline was $\sim 150 \mathrm{~m}$, providing an angular resolution $>9^{\prime \prime}$ at $7 \mathrm{~mm}$ and $>4^{\prime \prime}$ at $3 \mathrm{~mm}$. The continuum emission (the $1^{\prime \prime}$ Einstein

\footnotetext{
1 All velocities throughout this work refer to the heliocentric reference frame, adopting a redshift $z=0.88582$.
} 
Table 1. Summary of the observations.

\begin{tabular}{lcccccccc}
\hline \hline Date & Telescope & Band & $\begin{array}{c}\text { Frequency } \\
(\mathrm{GHz})\end{array}$ & $\begin{array}{c}\delta \mathrm{V}^{a} \\
\left(\mathrm{~km} \mathrm{~s}^{-1}\right)\end{array}$ & $\begin{array}{c}\sigma^{b} \\
\left(10^{-3}\right)\end{array}$ & $I_{\mathrm{bg}}(\mathrm{SW})^{c}$ & $R^{d}$ & Notes \\
\hline 2011 Jul. 24 & PdBI & $3 \mathrm{~mm}$ & $93.0-96.6$ & $-/ 2.0^{e}$ & $-/ 2.3^{e}$ & $0.416 \pm 0.006$ & $1.40 \pm 0.03$ & Total flux $=2.6 \mathrm{Jy}$ \\
2011 Jul. 27 & ATCA & $7 \mathrm{~mm}$ & $44.8-48.9$ & 6.3 & 2.0 & $0.402 \pm 0.003$ & $1.49 \pm 0.01$ & \\
2011 Jul. 27 & ATCA & $3 \mathrm{~mm}$ & $90.8-96.4$ & 3.2 & 2.8 & $0.397 \pm 0.004$ & $1.52 \pm 0.02$ & Total flux $=2.1 \mathrm{Jy}$ \\
2011 Aug. 17 & PdBI & $2 \mathrm{~mm}$ & $140.2-143.8$ & $4.1 / 1.3^{e}$ & $4.5 / 6.8^{e}$ & $0.437 \pm 0.010$ & $1.29 \pm 0.05$ & Th \\
\hline
\end{tabular}

Notes. ${ }^{(a)}$ Velocity resolution for the $\mathrm{HCO}^{+}$lines. ${ }^{(b)}$ Noise level normalized to the total continuum (NE+SW images). ${ }^{(c)}$ Fraction of the total continuum intensity corresponding to the SW image, measured on the saturated part of the spectra between $-7 \mathrm{~km} \mathrm{~s}^{-1}$ and $+7 \mathrm{~km} \mathrm{~s}^{-1}$. (d) Magnification factor $R=I_{\mathrm{bg}}(\mathrm{NE}) / I_{\mathrm{bg}}(\mathrm{SW})$, assuming no flux arises from other components. ${ }^{(e)}$ Corresponding to the WIDEX and narrow-band correlator output, respectively.

ring and NE/SW images) of PKS 1830-211 was therefore not resolved.

The CABB correlator was set up to provide a bandwidth of $2 \mathrm{GHz}$ with a spectral channel spacing of $1 \mathrm{MHz}$ in each sideband. We used two partly overlapping tunings at $7 \mathrm{~mm}$ and three at $3 \mathrm{~mm}$ to cover the frequency ranges given in Table 1 . The on-source integration time was $0.5 \mathrm{~h} /$ tuning at $7 \mathrm{~mm}$ and $1.5 \mathrm{~h} /$ tuning at $3 \mathrm{~mm}$. The total allocated time, including calibration and overheads, was $8 \mathrm{~h}$. The receivers were tuned in dual polarization mode. Both polarizations were averaged after the data calibration. The bandpass calibration was performed on the bright quasar 1921-293 for each tuning.

The data reduction was done using MIRIAD ${ }^{2}$ (Sault et al. 1995). Source visibilities were self-calibrated and the (unresolved) continuum level normalized to unity. Spectra were then extracted from the calibrated visibilities following the same method as described by Muller et al. (2011). A fixed Doppler track correction of $+13 \mathrm{~km} \mathrm{~s}^{-1}$ was applied to all data. Since the drift in velocity is less than $0.1 \mathrm{~km} \mathrm{~s}^{-1} / \mathrm{h}$, the effect introduced by adopting a fixed correction is negligible in comparison to the spectral resolution.

In complement to this dataset, we use previous ATCA $7 \mathrm{~mm}$ observations from 2009 September 1 and 2 published in Muller et al. (2011), specifically for transitions of $\mathrm{c}-\mathrm{C}_{3} \mathrm{H}_{2}, \mathrm{CH}_{3} \mathrm{CN}$, SO, and $\mathrm{HC}_{3} \mathrm{~N}$ (see Sect. 3.3.1). These observations were obtained and reduced following the same procedure as described above.

\subsection{Plateau de Bure interferometer (PdBI)}

Observations were obtained with the PdBI on 2011 July 24 at $3 \mathrm{~mm}$, and on 2011 August 17 at $2 \mathrm{~mm}$, with five antennas in a compact array. All baselines were shorter than $100 \mathrm{~m}$, so that the continuum emission of PKS 1830-211 was not resolved, as per the ATCA observations.

The wide-band correlator WIDEX was used, providing a 3.6 GHz bandwidth with a spectral resolution of $1.95 \mathrm{MHz}$. In addition, the narrow-band correlator was configured to select two $160 \mathrm{MHz}$ spectral bands in order to provide a finer spectral resolution of $0.625 \mathrm{MHz}$, with dual polarization. At $3 \mathrm{~mm}$, these narrow bands were set to cover the $0 \mathrm{~km} \mathrm{~s}^{-1}$ and $-147 \mathrm{~km} \mathrm{~s}^{-1}$ absorption lines of both $\mathrm{HCO}^{+}$and $\mathrm{HCN} \mathrm{2-1.} \mathrm{At} 2 \mathrm{~mm}$, the narrow-band IF range allowed us to cover only the $0 \mathrm{~km} \mathrm{~s}^{-1}$ and $-147 \mathrm{~km} \mathrm{~s}^{-1}$ components of the $\mathrm{HCO}^{+} 3-2$ line and the $-147 \mathrm{~km} \mathrm{~s}^{-1}$ component of the HCN 3-2 line (see the spectra in Fig. 1).

The bandpass was calibrated on the bright quasars $1749+$ 096 (at $3 \mathrm{~mm}$ ) and 3C454.3 (at $2 \mathrm{~mm}$ ). We followed the standard

\footnotetext{
2 http://www.atnf.csiro.au/computing/software/miriad/
}

calibration procedure in $\mathrm{CLIC}^{3}$, using the "Self-Calibration on point source" routine. The spectra were then exported to $\mathrm{CLASS}^{2}$, where we divided the spectral baseline with polynomials of degree two for the narrow-band spectra, and degree 12 or 24 , depending on the case, for the full 3.6 GHz WIDEX bands, in order to correct for residual ripples in the spectral baseline. The target absorption lines are too narrow to be seriously affected by these polynomial orders.

Features corresponding to $\mathrm{O}_{3}$ atmospheric lines were identified in the final spectra. In particular, we flagged a region of $200 \mathrm{MHz}$ around the $142.175 \mathrm{GHz} \mathrm{O}_{3}$ line in the $2 \mathrm{~mm}$ data.

\subsection{Continuum illumination}

The absorption intensities $\left(I_{v}\right)$, measured from the total continuum level, are converted to optical depths $\left(\tau_{v}\right)$ according to:

$\tau_{v}=-\ln \left(1-\frac{I_{v}}{f_{\mathrm{c}} I_{\mathrm{bg}}}\right)$,

where $I_{\mathrm{bg}}$ and $f_{\mathrm{c}}$ are the fraction of the total continuum intensity (normalized to unity) and the continuum source covering factor, corresponding to each individual image of the quasar, respectively. We adopt a value $f_{\mathrm{c}}=1$ (see Wiklind \& Combes 1998; Muller \& Guélin 2008).

At radio wavelengths, the continuum emission of the background quasar is dominated by two bright and compact images to the NE and to the SW of the 1" Einstein ring (see Sect. 1.2). These NE and SW images are not resolved in our observations, and so we cannot measure their respective fluxes $I_{\mathrm{bg}}(\mathrm{NE})$ and $I_{\mathrm{bg}}(\mathrm{SW})$ directly. However, the $\mathrm{HCO}^{+}$and $\mathrm{HCN}$ lines appear to be flat-bottomed near $v=0 \mathrm{~km} \mathrm{~s}^{-1}$, with both retaining the same intensity for a given epoch (Fig. 1), which strongly suggests that they are saturated. The detection of the ${ }^{13} \mathrm{C}$ variants of these species (and even of other less abundant isotopologues), clearly implies that they are optically thick, with opacities higher than $\tau=10$. Because we know that the absorption near $v=0 \mathrm{~km} \mathrm{~s}^{-1}$ occurs in front of the SW image (Wiklind \& Combes 1998; Muller et al. 2006), we can measure $I_{\mathrm{bg}}(\mathrm{SW})$ from the saturation level of the $\mathrm{HCO}^{+}$and $\mathrm{HCN}$ lines between $v=-7 \mathrm{~km} \mathrm{~s}^{-1}$ and $v=+7 \mathrm{~km} \mathrm{~s}^{-1}$, under the assumption $f_{\mathrm{c}}=1$. The results are given in Table 1 . The comparison of the $\mathrm{HCO}^{+} J=3-2$ spectra obtained with the WIDEX correlator (velocity resolution $\delta V$ of $\left.\sim 4 \mathrm{~km} \mathrm{~s}^{-1}\right)$ and with the narrow-band correlator $\left(\delta V \sim 1 \mathrm{~km} \mathrm{~s}^{-1}\right)$ shows that the limited resolution in velocity does not significantly affect the derivation of the saturation level.

The measured values of $I_{\mathrm{bg}}(\mathrm{SW})$ differ between the three epochs, with the biggest change on the last measurement with the PdBI, obtained 20 days after the ATCA observations. As the

http://www.iram.fr/IRAMFR/GILDAS/ 


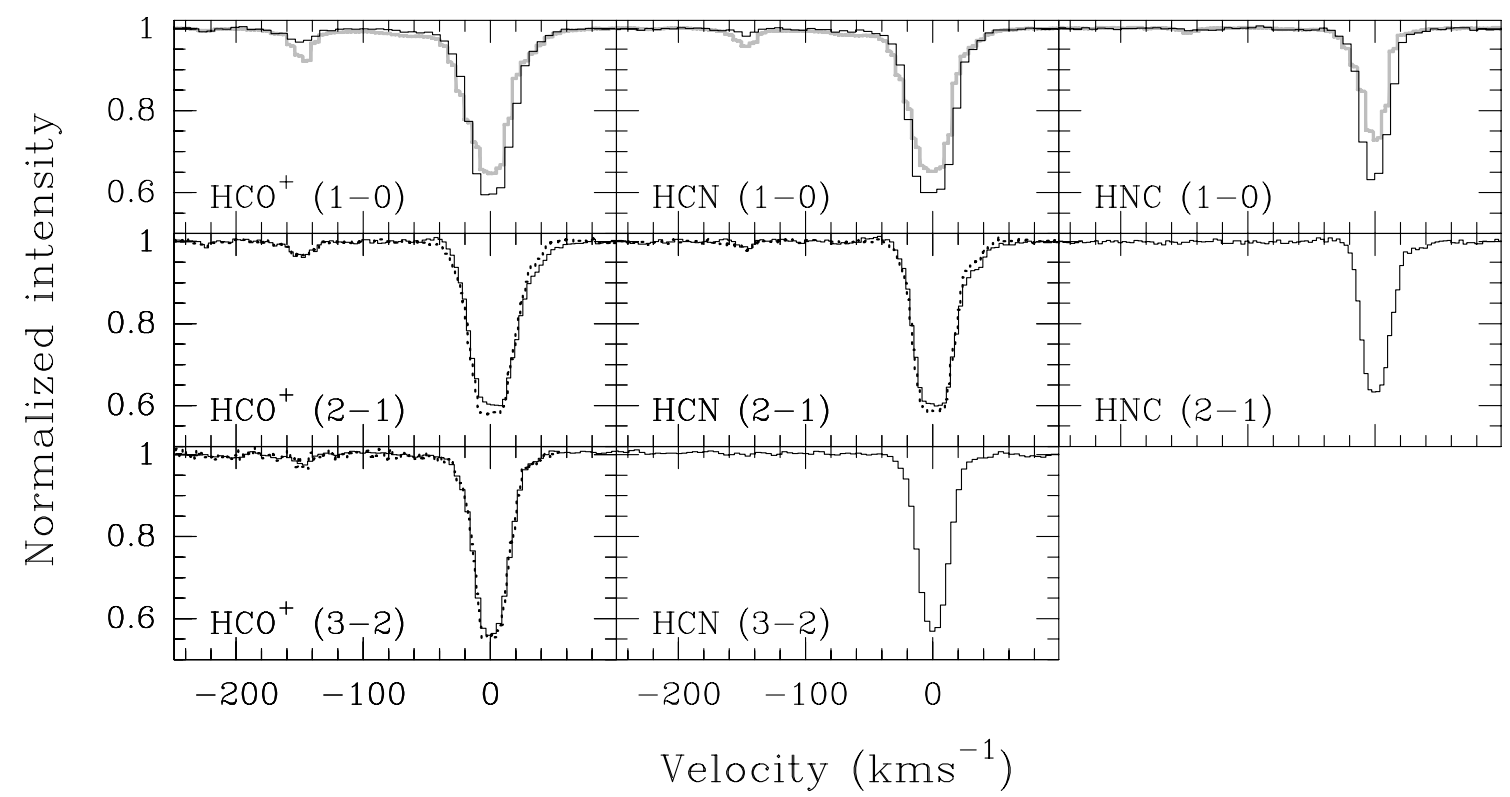

Fig. 1. Spectra of the strong absorption of $\mathrm{HCO}^{+}, \mathrm{HCN}$ and $\mathrm{HNC}$ transitions observed in 2011 with ATCA (full black line). The dotted spectra correspond to the higher spectral resolution PdBI observations with the narrow-band correlator (see Table 1 ). The $J=1-0$ spectra observed in our previous ATCA $7 \mathrm{~mm}$ survey (Muller et al. 2011) are superimposed in light grey. The intensity is normalized to the total continuum flux (NE plus SW images).

saturation level does not depend up the absolute flux density calibration (the intensities are normalized to the total continuum level), we believe that these changes are significant and not of instrumental origin. They could be caused either by a change of the NE/SW ratio due to the time delay of $\sim 25 \mathrm{~d}$ between the two images of the quasar (Lovell et al. 1998; Wiklind \& Combes 1999), or by a change of the source covering factor if the sightline moved between the observations. Changes of the continuum morphology, related to the jet activity of the blazar, are clearly seen within a time span of two weeks (Jin et al. 2003; see also Garrett et al. 1997), but monitoring on a shorter timescale is still lacking, to the best of our knowledge.

The changes seen between July and August 2011 are unfortunate for our purpose, because the lines of sight through the intervening galaxy - hence the column density and physical conditions of the absorbing gas - might have changed. This emphasizes the need to conduct the observations within a short time interval when the goal is to combine different observations toward PKS 1830-211. In the remainder of the paper, we will treat only the ATCA observations of the lines corresponding to the SW component, and assume that no significant intra-day variations occur.

In order to take into account the uncertainty in the continuum illumination $\Delta I_{\mathrm{bg}}$, we have added quadratically to the fit uncertainties of the integrated opacities the quantity:

$\Delta\left(\int \tau \mathrm{d} v\right)=\left(\int \tau \mathrm{d} v\right) \times \frac{\Delta I_{\mathrm{bg}}}{I_{\mathrm{bg}}} \times F\left(I / I_{\mathrm{bg}}\right)$,

where the function $F(x)=x /((x-1) \ln (1-x))$, is obtained from the differentiation of Eq. (1) with respect to $I_{\mathrm{bg}}$. This correction is simply equivalent to $\frac{\Delta \tau}{\tau}=\frac{\Delta I_{\mathrm{bg}}}{I_{\mathrm{bg}}}$ for optically thin lines.

The magnification ratios $R=\mathrm{NE} / \mathrm{SW}$, which we take as the fraction $\left(1-I_{\mathrm{bg}}(\mathrm{SW})\right) / I_{\mathrm{bg}}(\mathrm{SW})$, are listed in Table 1 . They are within the range of values compiled by Muller \& Guélin (2008), using the same measurement method of the saturation level of the $\mathrm{HCO}^{+} \mathrm{SW}$ absorption.

\section{Analysis}

In this section we describe the analysis of the spectral data, starting from a simple approach used to derive rotation temperatures. We then investigate the effects of local physical conditions on the line excitation. Finally, we provide a global solution of the problem using a Monte-Carlo Markov chain (MCMC) method, from which we derive our final measurement of $T_{\mathrm{CMB}}$ at $z=0.89$.

\subsection{Rotation temperatures}

First, we focus on deriving the rotation temperature of the observed species, assuming that the excitation is dominated by radiative coupling with the CMB (low-density case). Under this condition, the population of the energy levels follows a Boltzmann distribution, for which the rotation temperature equals $T_{\mathrm{CMB}}$. The rotation temperature $T_{\text {rot }}$ between two energy levels (upper $u$ and lower $l$ ) is defined by the Boltzmann equation:

$\frac{n_{u}}{n_{l}}=\frac{g_{u}}{g_{l}} \exp \left(-\frac{h v_{u l}}{k T_{\text {rot }}}\right)$

where $n_{u}$ and $n_{l}$ are the populations, and $g_{u}$ and $g_{l}$ the degeneracies of the upper and lower level, respectively, connected by a transition at frequency $v_{u l}$. The column density $\mathrm{N}_{\mathrm{LTE}}{ }^{4}$ can then be derived from:

$N_{\mathrm{LTE}}=\frac{3 h}{8 \pi^{3} \mu^{2} S_{u l}} \frac{Q\left(T_{\mathrm{rot}}\right) \exp \left(\frac{E_{l}}{k_{\mathrm{B}} T_{\mathrm{rot}}}\right)}{\left[1-\exp \left(-\frac{h v}{k_{\mathrm{B}} T_{\mathrm{rot}}}\right)\right]} \int \tau \mathrm{d} v$,

where $Q\left(T_{\text {rot }}\right)$ is the partition function, $E_{l}$ the energy of the lower level with respect to ground state, $\mu$ the dipole moment, $v$ is the

4 We refer to this estimate as the LTE column density, although strictly speaking, we simply assume that the excitation is dominated by the CMB photons. 

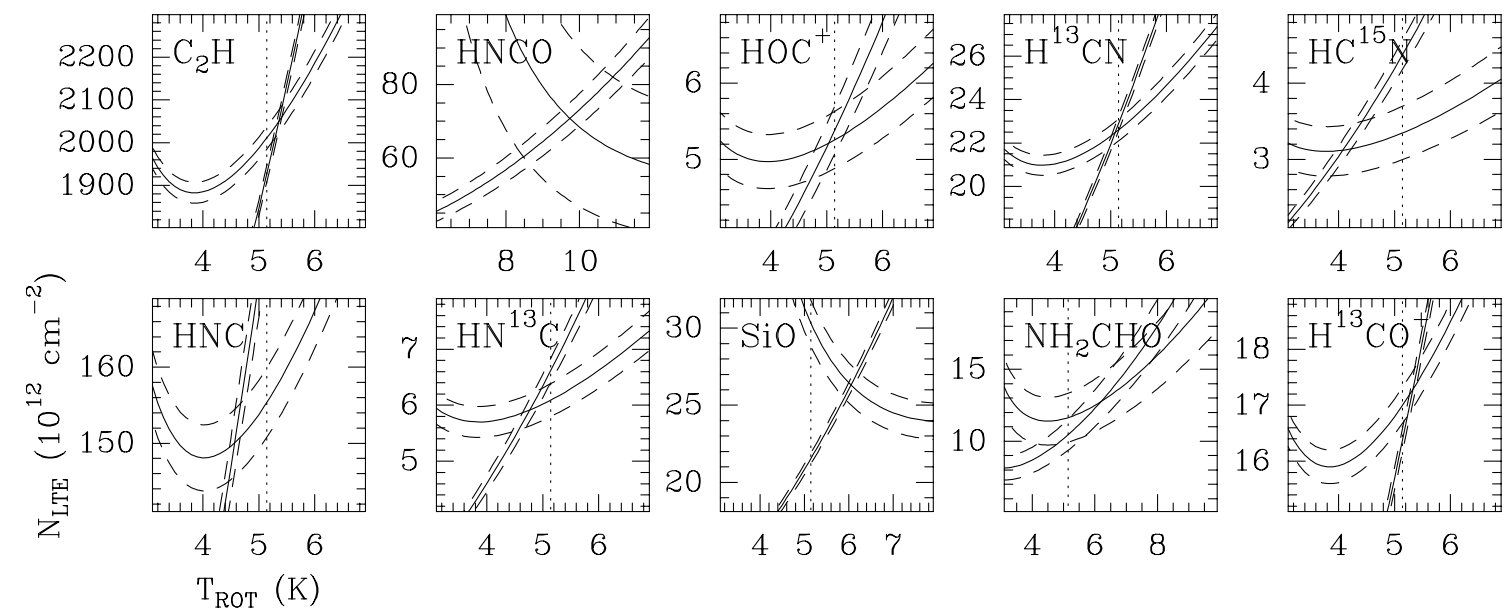

Fig. 2. Evolution of the column density $\left(N_{\mathrm{LTE}}\right)$ as a function of the rotation temperature $\left(T_{\text {rot }}\right)$ for the transitions of the molecular species observed in 2011 (Table A.1 and Eq. (4)). Each curve corresponds to a different transition and the dashed curves delimit the $1 \sigma$ uncertainties. The curve intersections give the values of $N_{\text {col }}$ and $T_{\text {rot }}$. The vertical dotted line indicates $T_{\mathrm{CMB}}=5.14 \mathrm{~K}$.

frequency, $S_{u l}$ the line strength and $\int \tau \mathrm{d} v$ the integrated opacity of the line.

The kinetic temperature of the gas along the SW line of sight is on the order of $\sim 80 \mathrm{~K}$, as indicated by observations of the symmetric top molecules $\mathrm{NH}_{3}$ and $\mathrm{CH}_{3} \mathrm{CN}$ (Henkel et al. 2008; Muller et al. 2011). Because the density is moderate (on the order of $10^{3} \mathrm{~cm}^{-3}$, Henkel et al. 2009), the gas is sub-thermally excited with the excitation mostly coupled radiatively with the CMB. Hence, the rotation temperatures are expected to be close to (only slightly higher than, see Sect. 3.2) $T_{\mathrm{CMB}}$. The rotation temperatures of several species as derived by Combes \& Wiklind (1999), Menten et al. (1999), Henkel et al. (2009), and Muller et al. (2011) in the SW line of sight are consistent with this picture.

We note that the Rayleigh-Jeans approximation does not hold for $v>100 \mathrm{GHz}$ transitions when the temperature is low $(\sim 5 \mathrm{~K})$, and the rotation diagram method (see Muller et al. 2011, for lower frequency data) is not applicable to derive the rotation temperature in this case. Instead, we use Eq. (4), and plot the $N_{\text {LTE }}-T_{\text {rot }}$ curves for the different observed transitions (Fig. 2). These curves have slightly different sensitivities to the temperature and their intersection gives the rotation temperature and column density. The results are given in Table 2 and, as expected, the rotation temperatures are all close to $5 \mathrm{~K}$.

\subsection{Effects of local physical conditions}

In order to test the impact of the local physical conditions on the line excitation, we have run models using the non-LTE molecular radiative transfer code RADEX (van der Tak et al. 2007), varying parameters such as the kinetic temperature $\left(T_{\text {kin }}\right)$, the $\mathrm{H}_{2}$ density $\left(n_{\mathrm{H}_{2}}\right)$, and the local radiation field.

RADEX uses the escape probability method for a homogeneous medium and is based on radiative and collisional transition rates, which are only available for a few species in a limited range of temperatures and column densities. In this work, we use the standard files available from the Leiden Atomic and Molecular Database ${ }^{5}$ (LAMDA, Schöier et al. 2005), except for $\mathrm{HC}_{3} \mathrm{~N}$, for which rates extended to $100 \mathrm{~K}$ were kindly provided to us by Faure (Wernli et al. 2007a,b; and Faure \& Wiesenfeld, in prep.). We consider that $\mathrm{H}_{2}$ molecules are the main collision partners.

\footnotetext{
5 http://home.strw.leidenuniv.nl/ moldata/
}

Table 2. Results for rotation temperature measurements toward the SW absorption.

\begin{tabular}{lccc}
\hline \hline Species & $\begin{array}{c}\text { Dipole } \\
\text { moment } \\
\text { (Debye) }\end{array}$ & $\begin{array}{c}\text { Date } \\
\text { of the } \\
\text { observations }\end{array}$ & $\begin{array}{c}T_{\text {rot }} \\
(\mathrm{K})\end{array}$ \\
\hline $\mathrm{C}_{2} \mathrm{H}$ & 0.77 & 2011 & $5.3 \pm 0.1$ \\
$\mathrm{SO}$ & 1.54 & 2009 & $5.4 \pm 1.4$ \\
$\mathrm{HNCO}$ & 1.58 & 2011 & $9.8 \pm 1.5$ \\
$\mathrm{HOC}$ & 2.77 & 2011 & $5.1 \pm 0.4$ \\
$\mathrm{H}^{13} \mathrm{CN}$ & 2.99 & 2011 & $5.1 \pm 0.2$ \\
$\mathrm{HC}^{15} \mathrm{~N}$ & 2.99 & 2011 & $4.1 \pm 0.4$ \\
$\mathrm{HNC}$ & 3.05 & 2011 & $4.6 \pm 0.2^{\dagger}$ \\
$\mathrm{HN}{ }^{13} \mathrm{C}$ & 3.05 & 2011 & $4.8 \pm 0.3$ \\
$\mathrm{SiO}$ & 3.10 & 2011 & $6.0 \pm 0.2$ \\
${\mathrm{c}-\mathrm{C}_{3} \mathrm{H}_{2}-\mathrm{O}}^{+}$ & 3.43 & 2009 & $5.6 \pm 0.4$ \\
$\mathrm{c}^{-} \mathrm{C}_{3} \mathrm{H}_{2}-\mathrm{p}$ & 3.43 & 2009 & $5.4 \pm 1.0$ \\
$\mathrm{HC}_{3} \mathrm{~N}$ & 3.73 & 2009 & $6.3 \pm 1.3$ \\
$\mathrm{H}^{13} \mathrm{CO}^{+}$ & 3.90 & 2011 & $5.3 \pm 0.1$ \\
\hline
\end{tabular}

Notes. ${ }^{(\dagger)}$ Might be affected by opacity effects. Uncertainties are given at $1 \sigma$.

\subsection{1. $\mathrm{H}_{2}$ density and kinetic temperature}

We have run RADEX simulations varying $n_{\mathrm{H}_{2}}$ between 500 and $10^{4} \mathrm{~cm}^{-3}$ for two representative values of the kinetic temperature $T_{\text {kin }}=50 \mathrm{~K}$ and $100 \mathrm{~K}$. These ranges encompass previous estimates of the density ( $2000 \mathrm{~cm}^{-3}$, Henkel et al. 2009), and of the kinetic temperature $(\sim 80 \mathrm{~K}$, Henkel et al. 2008$)$ toward the SW component of PKS 1830-211. Such a value of the kinetic temperature is typical of $T_{\text {kin }}$ observed in Galactic diffuse clouds (e.g. Snow \& McCall 2006). At this stage, and for sake of simplicity, we have set the column densities of the various species to the values that can be derived directly from the intersection of the $N_{\text {LTE }}$ vs. $T_{\text {rot }}$ curves (Fig. 2).

The results of our RADEX simulations can be seen in Fig. 3, where the curves indicate the evolution of the rotation temperature for a given transition as a function of the $\mathrm{H}_{2}$ density, for $\mathrm{HCO}^{+}, \mathrm{HCN}, \mathrm{HNC}$, some of their isotopologues, $\mathrm{HC}_{3} \mathrm{~N}, \mathrm{SO}$, $\mathrm{HNCO}, \mathrm{SiO}$, and $\mathrm{c}-\mathrm{C}_{3} \mathrm{H}_{2}$ (ortho and para forms). The rotation temperature measured from the observation of two transitions is the average of the curves corresponding to these two transitions. The rotation temperature increases with $\mathrm{H}_{2}$ density, as 


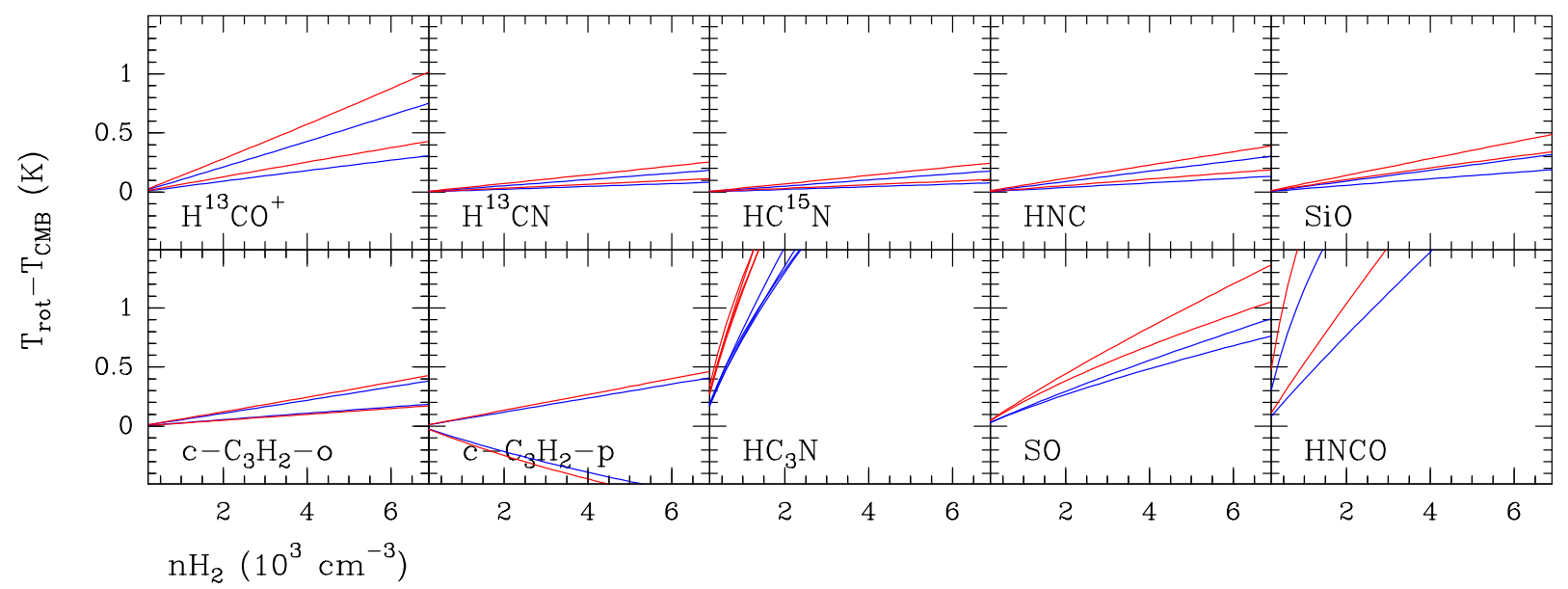

Fig. 3. RADEX predictions of the rotation temperatures as a function of $\mathrm{H}_{2}$ density for the transitions of $\mathrm{H}^{13} \mathrm{CO}^{+}, \mathrm{H}^{13} \mathrm{CN}, \mathrm{HC}{ }^{15} \mathrm{~N}, \mathrm{HNC}, \mathrm{SiO}$, and HNCO (listed in Table A.1), and transitions of $\mathrm{c}-\mathrm{C}_{3} \mathrm{H}_{2}, 1-\mathrm{C}_{3} \mathrm{H}_{2}, \mathrm{SO}$, and $\mathrm{HC}_{3} \mathrm{~N}$ (listed in Table A.1 by Muller et al. 2011). We assume $T_{\mathrm{CMB}}=$ $5.14 \mathrm{~K}, T_{\text {kin }}=50 \mathrm{~K}$ (blue), and $T_{\text {kin }}=100 \mathrm{~K}$ (red). The rotation temperature derived from the observations of two (or more) transitions is the average of the corresponding rotation temperatures curves.

can be expected when collisional excitation progressively adds to the radiative excitation from the CMB. This is the case of all transitions, except for the $\mathrm{c}-\mathrm{C}_{3} \mathrm{H}_{2}$ (para) $33_{31}-3_{22}$, which shows a decrease of $T_{\text {rot }}$ with $n_{\mathrm{H}_{2}}$.

Among the various species investigated here, the most sensitive to collisional excitation are $\mathrm{HC}_{3} \mathrm{~N}$ and $\mathrm{HNCO}$, for which $\Delta T=T_{\text {rot }}-T_{\mathrm{CMB}}$ reaches values $>1 \mathrm{~K}$ for $n_{\mathrm{H}_{2}}>2000 \mathrm{~cm}^{-3}$. On the other hand, the transitions of $\mathrm{HCN}, \mathrm{HNC}$ and $\mathrm{c}-\mathrm{C}_{3} \mathrm{H}_{2}$ (ortho) (and their isotopic variants when applicable) have $\Delta T$ of less than a few tenths of a Kelvin for $n_{\mathrm{H}_{2}} \sim 2000 \mathrm{~cm}^{-3}$, and can therefore be considered as reliable $T_{\mathrm{CMB}}$ indicators.

\subsubsection{Local radiation field}

We now add the excitation from a local radiation field. We consider first the mean background radiation near the Sun's location in the Galaxy, which is due mainly to dust thermal emission (we adopt a single-temperature component with $T=23 \mathrm{~K}$, Wright et al. 1991). Non-thermal radiation is negligible at $\mathrm{mm} / \mathrm{submm}$ wavelengths. We did not find any measurable differences from the case with CMB excitation only. Gradually increasing the dust temperature, we start to see some effects on the rotation temperature of $\mathrm{HNCO}$ for $T_{\text {dust }} \sim 100 \mathrm{~K}$, while no other molecules exhibit any change. We thus conclude that the effect of the local radiation on the excitation of the observed lines is negligible, unless very peculiar conditions prevail.

\subsection{A global solution for the excitation conditions with Monte-Carlo Markov chains}

In the following, we adopt a global method for simultaneously solving the physical conditions and $T_{\mathrm{CMB}}$. We first describe our working assumptions and the RADEX modeling, and then the Monte-Carlo Markov chain approach used to adjust the model to the spectra.

\subsubsection{RADEX model}

For a given molecular species, we use RADEX to compute the velocity integrated intensity of each line present in our spectra, given the background radiation field $\left(T_{\mathrm{CMB}}\right)$, the kinetic temperature $\left(T_{\text {kin }}\right)$, the number density of $\mathrm{H}_{2}$ molecules $\left(n_{\mathrm{H}_{2}}\right)$, the column density of each molecule $\left(N_{\mathrm{col}}\right)$ and the width of the molecular line $(\Delta V)$, which is assumed to be Gaussian in profile.

The physical conditions could vary with time (whenever the barycenter of the continuum emission drifts). We therefore consider independently the physical condition for the 2009 and 2011 data. For simplicity, we assume that the absorbing gas is homogeneous and can be characterized, for each epochs, by a single specific kinetic temperature $T_{\text {kin }}$ and $n_{\mathrm{H}_{2}}$ density, common for all molecular species. For the SW line of sight, we thus have the set of parameters $T_{\mathrm{kin}} / n_{\mathrm{H}_{2}}(2009)$ and $T_{\mathrm{kin}} / n_{\mathrm{H}_{2}}(2011)$. The background radiation field is assumed to be constant and equal to the CMB.

Given the velocity centroid $\left(V_{0}\right)$ and linewidth $(\Delta V)$, specific for each molecular species, we are then able to construct a synthetic spectrum from Eq. (1), which reproduces the observed frequencies. For each species, we thus have three free parameters $\left(N_{\mathrm{col}}, \Delta V\right.$, and $\left.V_{0}\right)$ and three tied parameters ( $T_{\mathrm{kin}}, n_{\mathrm{H}_{2}}$, and $T_{\mathrm{CMB}}$ ) to fit. We include all species with molecular data available in the LAMDA database, and with at least two, non saturated, lines in the observed spectra. For this we used: $\mathrm{H}^{13} \mathrm{CO}^{+}, \mathrm{H}^{13} \mathrm{CN}, \mathrm{HC}^{15} \mathrm{~N}, \mathrm{c}-\mathrm{C}_{3} \mathrm{H}_{2}$ (both ortho and para forms), $\mathrm{SiO}, \mathrm{CH}_{3} \mathrm{CN}, \mathrm{SO}, \mathrm{HC}_{3} \mathrm{~N}$, and $\mathrm{HNCO}$.

\subsubsection{Adjusting the model}

This high number of variables is very difficult to constrain by using classical techniques. We therefore adopt a MCMC method, widely used to estimate cosmological parameters (e.g. Dunkley et al. 2005 and references therein). We use a subclass of the MCMC methods, the Metropolis-Hastings algorithm to probe the probability distribution of our problem and study the degeneracies between the different parameters.

For each parameter, we choose the proposed density, or jumping distribution, as Gaussian distributions, with variances set initially to the result of the estimate made in Muller et al. (2011). As the collisional rates are only known for a limited temperature and density range, we reject all jumps going outside this. The likelihood is simply computed by $p \propto \exp \left(-\chi^{2} / 2\right)$, with

$\chi^{2}=\sum_{v=1}^{N} \frac{\left(s_{v}-s_{v, \bmod }\right)^{2}}{\sigma_{v}^{2}+\sigma_{v, \bmod }^{2}}$, 
Table 3. Results (median and 68 percentile error) of the Metropolis-Hasting estimates of the physical conditions toward the SW component of the 2009 and 2011 campaigns.

\begin{tabular}{|c|c|c|c|c|c|c|}
\hline Species & $\begin{array}{c}T_{\mathrm{CMB}} \\
{[\mathrm{K}]}\end{array}$ & $\begin{array}{c}n_{\mathrm{H}_{2}} \\
{\left[\mathrm{~cm}^{-3}\right]}\end{array}$ & $\begin{array}{l}T_{\text {kin }} \\
{[\mathrm{K}]}\end{array}$ & $\begin{array}{c}\log N_{\text {col }} \\
{\left[\mathrm{cm}^{-2}\right]}\end{array}$ & $\begin{array}{c}\Delta V \\
{\left[\mathrm{~km} \mathrm{~s}^{-1}\right]}\end{array}$ & $\begin{array}{c}V_{0} \\
{\left[\mathrm{~km} \mathrm{~s}^{-1}\right]}\end{array}$ \\
\hline \multicolumn{7}{|c|}{$5.08_{-0.10}^{+0.10}$} \\
\hline (2009) & - & $800_{-400}^{+400}$ & $81_{-7}^{+8}$ & & & \\
\hline $\mathrm{CH}_{3} \mathrm{CN}$ & - & - & - & $13.200_{-0.017}^{+0.016}$ & $20.0_{-0.9}^{+0.9}$ & $-2.3_{-0.4}^{+0.4}$ \\
\hline SO & - & - & - & $13.458_{-0.023}^{+0.023}$ & $19.8_{-0.9}^{+0.9}$ & $-1.8_{-0.4}^{+0.4}$ \\
\hline $\mathrm{c}-\mathrm{C}_{3} \mathrm{H}_{2}(\mathrm{o})$ & - & - & - & $13.711_{-0.016}^{+0.016}$ & $24.1_{-0.6}^{+0.6}$ & $-3.1_{-0.3}^{+0.3}$ \\
\hline $\mathrm{c}-\mathrm{C}_{3} \mathrm{H}_{2}(\mathrm{p})$ & - & - & - & $13.223_{-0.019}^{+0.019}$ & $21.0_{-0.7}^{+0.7}$ & $-1.8_{-0.3}^{+0.3}$ \\
\hline $\mathrm{HC}_{3} \mathrm{~N}$ & - & - & - & $13.037_{-0.025}^{+0.027}$ & $22.3_{-1.1}^{+1.2}$ & $-5.6_{-0.5}^{+0.5}$ \\
\hline (2011) & - & $2200_{-900}^{+900}$ & $82_{-9}^{+11}$ & & & \\
\hline $\mathrm{CH}_{3} \mathrm{CN}$ & - & - & - & $13.530_{-0.024}^{+0.024}$ & $14.6_{-1.0}^{+1.1}$ & $-3.9_{-0.5}^{+0.5}$ \\
\hline $\mathrm{H}^{13} \mathrm{CO}^{+}$ & - & - & - & $13.264_{-0.008}^{+0.008}$ & $16.2_{-0.2}^{+0.2}$ & $-2.4_{-0.1}^{+0.1}$ \\
\hline $\mathrm{H}^{13} \mathrm{CN}$ & - & - & - & $13.385_{-0.010}^{+0.010}$ & $17.0_{-0.2}^{+0.2}$ & $-2.8_{-0.1}^{+0.1}$ \\
\hline $\mathrm{HC}^{15} \mathrm{~N}$ & - & - & - & $12.6077_{-0.024}^{+0.023}$ & $14.2_{-0.8}^{+0.9}$ & $-3.2_{-0.4}^{+0.4}$ \\
\hline $\mathrm{HNCO}$ & - & - & - & $13.662_{-0.030}^{+0.029}$ & $14.5_{-1.2}^{+1.2}$ & $-4.6_{-0.4}^{+0.4}$ \\
\hline $\mathrm{SiO}$ & - & - & - & $13.369_{-0.009}^{+0.009}$ & $14.6_{-0.3}^{+0.3}$ & $-3.0_{-0.1}^{+0.1}$ \\
\hline
\end{tabular}

Notes. $N_{\text {col }}, \Delta V$, and $V_{0}$ are the column densities, linewidths (FWHM), and velocity centroids, respectively.

where $s_{v}$ is the observed spectrum with its uncertainties $\sigma_{v}$ at frequency channel $v$, and $s_{v, \text { mod }}$ the modeled spectra with its uncertainty $\sigma_{v \text {,mod }}$ in order to take into account the uncertainty on the continuum illumination $\Delta I_{\text {bg }}$ (see Eq. (2)).

In order to speed up the convergence of the adjustment after a few initial chains, the variances were set according to the results of the chains themselves. Note that the final result does not depend on the proposed density distribution, as long as it is symmetric and the chains have converged. We used the spectral analysis described in Dunkley et al. (2005) to ensure the convergence of our chains before doing the analysis.

\subsubsection{Result of the Monte-Carlo Markov chain method}

One major advantage of the MCMC approach is that it allows us to take all uncertainties into account when deriving the posterior distribution of the parameters. Most of the parameters display a posterior Gaussian distribution with median value and 68 percentile error reported in Table 3. Figure 4 presents the results for a subset of the parameters, $T_{\mathrm{CMB}}$ and, for the two observing campaigns, $T_{\text {kin }}$ and $n_{\mathrm{H}_{2}}$. As expected for both campaigns, there is a slight degeneracy between $T_{\mathrm{CMB}}$ and $n_{\mathrm{H}_{2}}$, where a higher background radiation field could be compensated by a lower $\mathrm{H}_{2}$ density. However, the number of lines used here allows us to partially remove this degeneracy, puting firm constraints on $T_{\mathrm{CMB}}$. A percentile analysis (68\% confidence level) of the final chains, including all lines from all species for both campaigns, leads to a value of the CMB temperature of

$T_{\mathrm{CMB}}(z=0.89)=5.0791_{-0.0994}^{+0.0993} \mathrm{~K}$.

\section{Discussion}

\subsection{Cosmological constraints}

Our measurement of $T_{\mathrm{CMB}}$ at $z=0.89$ is interesting because i) it is the most precise measurement at $z>0$ to date, reaching a remarkable precision of $2 \%$; ii) the observations of various molecular species allow us to constrain the physical conditions of the absorbing gas, therefore limiting a possible bias using only one species; and iii) it is derived at a redshift intermediate between $S-Z$ measurements in galaxy clusters $(z<0.6$ so far) and optical/UV quasar absorption systems $(z>1.5$ due to atmospheric cut-off), using an alternative method (molecular excitation).

Combining our value of the CMB temperature at $z=0.89$ with other results published in the literature (Fig. 5), we obtain a tighter constraint on the $T_{\mathrm{CMB}}(z)=T_{0} \times(1+z)^{(1-\alpha)}$ law, $\alpha=$ $+0.009 \pm 0.019$, compared to the constraint $\alpha=-0.007 \pm 0.027$ reported by Noterdaeme et al. (2011). Within the uncertainties, the CMB temperature evolution is consistent with the standard cosmology model, with adiabatic expansion and $\alpha=0$.

\subsection{Physical conditions of the absorbing gas toward the SW component}

Besides $T_{\mathrm{CMB}}$, our MCMC approach also allows us to constrain the physical conditions toward the SW component, independently for each of the 2009 and 2011 observations (Table 3). We find that the kinetic temperature is well constrained, mostly owing to the observations of different K-transitions of $\mathrm{CH}_{3} \mathrm{CN}$. Our result of $T_{\text {kin }} \sim 80 \mathrm{~K}$ is comparable for both epochs and similar to the value derived by Henkel et al. (2008) from observations of $\mathrm{NH}_{3}$ inversion transitions in 2003-2004. Despite probable changes in the line of sight, it appears that the kinetic temperature of the absorbing gas remains unchanged. The situation is not so clear for the $\mathrm{H}_{2}$ volume density, which is not as well constrained as $T_{\text {kin }}$. From 2001-2002 data, Henkel et al. (2009) estimate $n_{\mathrm{H}_{2}} \sim 2000 \mathrm{~cm}^{-3}$, comparable to our $2011 \mathrm{re-}$ sult. By comparison, $n_{\mathrm{H}_{2}}$ for our 2009 data is roughly half that value, a result that is also found for the integrated opacities (or column density, all other parameters being equal) between 2011 and 2009. It is possible that the 2011 line of sight has intercepted a denser, more compact, absorbing component than in 2009. In any case, based on the observed rotation temperatures 


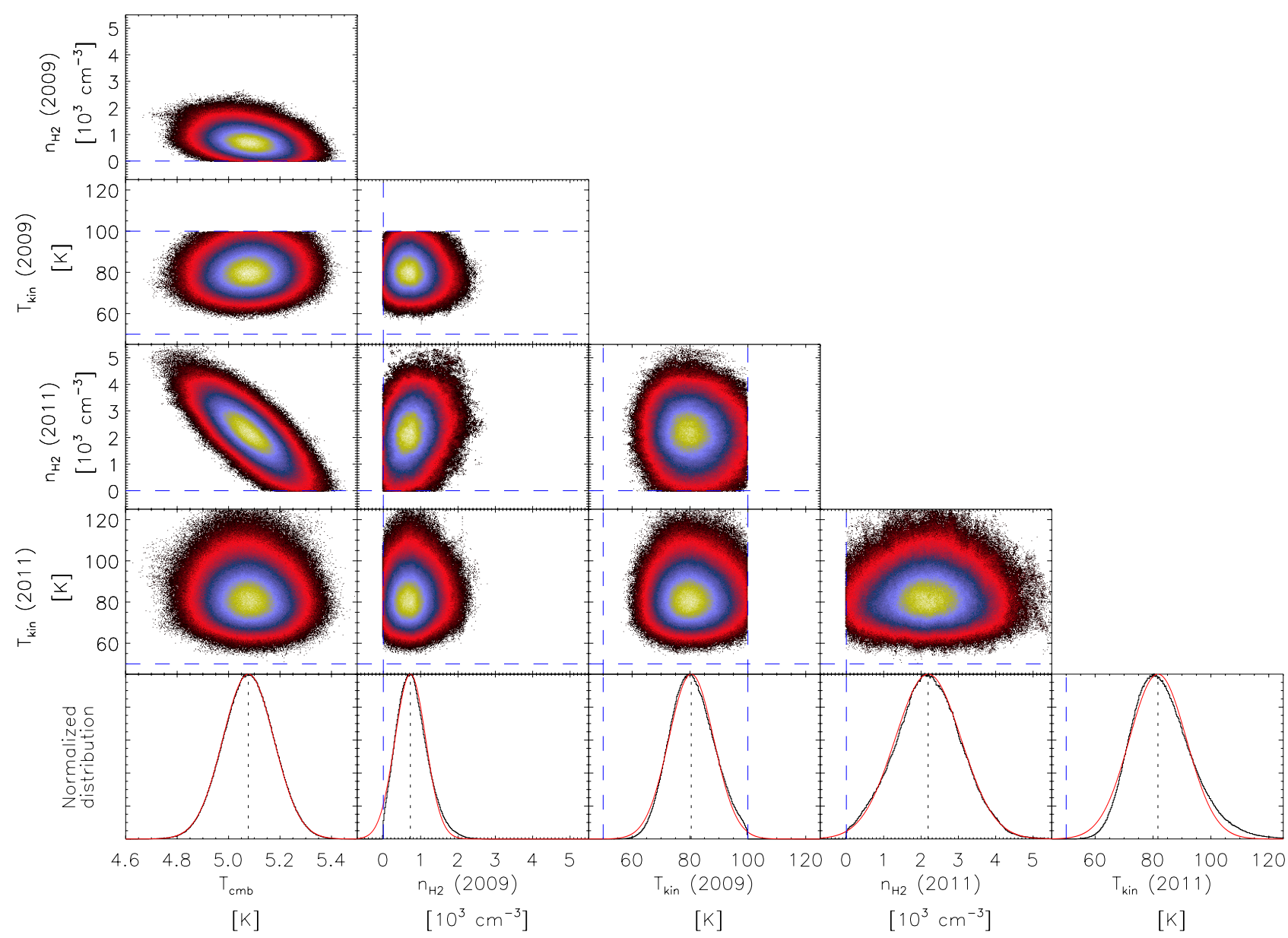

Fig. 4. One and two dimensional likelihood distributions for the Metropolis-Hasting estimates of the non-LTE condition toward the SW component of the 2009 and 2011 campaign. Only a subset of the parameters is represented. The blue dashed lines correspond to the common range of temperatures for which collisional rates are available in the LAMDA database for the considered set of species. The normalized 1D likelihood distributions (last row) are fitted by a Gaussian distribution (red continuous line).

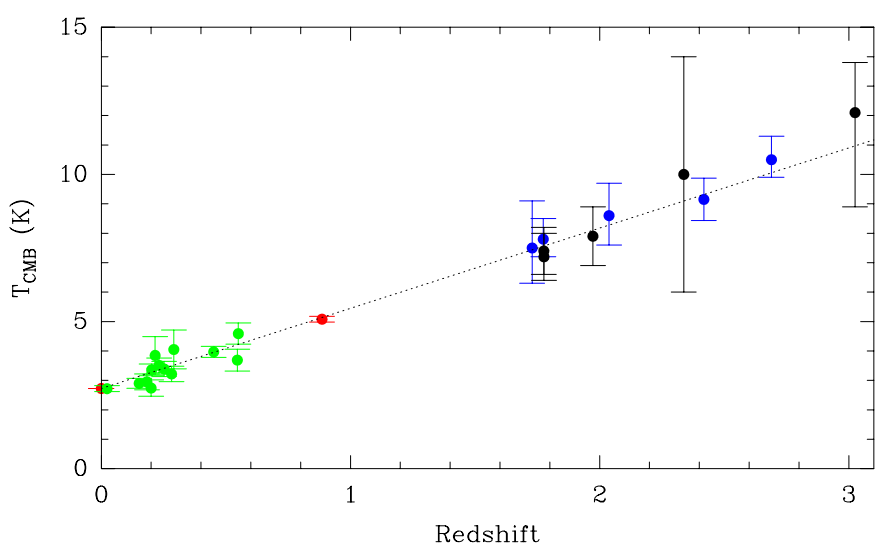

Fig. 5. Measurements of the CMB temperature as a function of redshift. Data points in green correspond to S-Z measurements toward galaxy clusters, in black to C I and C II absorption studies, in blue to $\mathrm{CO}$ absorption (see Noterdaeme et al. 2011 and references therein), with the value derived toward the PKS 1830-211 SW absorption marked in red (this paper). The dotted line corresponds to the law $T_{\mathrm{CMB}}=T_{0} \times(1+z)$.

and the value of $T_{\text {kin }}$, it is clear that the excitation of the absorbing gas is sub-thermal and that the density should be moderate $\left(<10^{4} \mathrm{~cm}^{-3}\right)$. This is consistent with the absorbing gas being composed of diffuse/translucent clouds (see Muller et al. 2011).

We note that the increase of absorption intensities in 2011 allows us to detect several new species toward this galaxy
(Table A.1): $\mathrm{NH}_{2} \mathrm{CHO}$ (2 transitions), ${ }^{30} \mathrm{SiO}(J=2-1), \mathrm{HCS}^{+}$ (whose $J=2-1$ transition shows up better out of the wing of the $\mathrm{c}-\mathrm{C}_{3} \mathrm{H}_{2} 2_{12}-1_{01}$ line than in the Muller et al. 2011 data) and, tentatively, $\mathrm{HOCO}^{+}$(detected at just $4 \sigma$ at rest frequency $85.531512 \mathrm{GHz}$ ).

\subsection{Limits and perspectives}

Our excitation analysis relies on the simplifying assumption of a homogeneous medium, characterized by single values of the $\mathrm{H}_{2}$ density and kinetic temperature common for all molecules, when it is an observational fact that Galactic clouds are highly structured and turbulent. Henkel et al. (2008) interpret their $\mathrm{NH}_{3}$ observations as two distinct components of absorbing gas, one at approximately $80 \mathrm{~K}$ occupying $80-90 \%$ of the column, and a second at kinetic temperature greater than $100 \mathrm{~K}$ occupying $10-20 \%$ of the column. The warmer component would be even more diffuse and more subthermally excited, if in pressure equilibrium with the denser and cooler phase. The typical scale length corresponding to a $\mathrm{H}_{2}$ column density of $2 \times 10^{22} \mathrm{~cm}^{-2}$ (see Sect. 4.2 by Muller et al. 2011 for the SW line of sight) and a density $n_{\mathrm{H}_{2}}=2000 \mathrm{~cm}^{-3}$ is $\sim 3 \mathrm{pc}$. Considering VLBI measurements of the size of the continuum images $(\sim 2 \mathrm{pc}$ in the image plane at $z=0.89$, Jin et al. 2003), the large covering factor of the absorbing clouds toward the SW line of sight and the time variation of the absorption profiles, we conclude that the extent of the clouds should be on the order of a few 
parsecs. Hence, the bulk of the absorption should be dominated by the cool component with $T_{\text {kin }} \sim 80 \mathrm{~K}$. We therefore suspect that the contribution from the warm component does not affect significantly our measurement of $T_{\mathrm{CMB}}$ and our hypothesis of an homogeneous medium is reasonable.

Although the derived value of the background radiation temperature is robust in our analysis, some neutral species like $\mathrm{SiO}$ and HNCO show slightly elevated rotation temperatures, suggesting that they may require higher densities than the ions. The simplest explanation is chemical stratification in the absorbing column, although some of the crucial collision rates are poorly known, especially when the ortho form of $\mathrm{H}_{2}$ is the main collision partner.

We also assume that the continuum source is fully intercepted by the absorber $\left(f_{\mathrm{c}}=1\right.$ in Eq. (1)). Nevertheless, this is not a critical assumption for optically thin lines and recent ALMA observations of the ground-state line of ortho water, resolving both NE and SW images of the quasar, show that the SW continuum source covering factor is indeed close to unity (Muller et al., in prep.), at least at the time of the observations in 2012.

Finally, we have considered $\mathrm{H}_{2}$ molecules only as collision partners. As shown by Liszt (2012) for the Galactic interstellar medium, molecular ions with large dipole moments, like $\mathrm{HCO}^{+}$, are most sensitive to collisional excitation by electrons. Electron-impact begins to compete with neutral collisions when the fractional ionization $n(e) / n_{\mathrm{H}} \gtrsim 10^{-4}$. The observed rotational temperatures of $\mathrm{H}^{13} \mathrm{CO}^{+}$and $\mathrm{HOC}^{+}$are both close to the expected $T_{\mathrm{CMB}}=5.14 \mathrm{~K}$, suggesting that the contribution from collisions with electrons is not significant.

The robustness of the $T_{\mathrm{CMB}}$ measurement toward PKS 1830-211 could be improved by investigating the different lines of sight to the lensed images of the background quasar. Observations of a stronger NE component (e.g. as in the period 1999-2003, see Muller \& Guélin 2008), with angular resolution sufficient to resolve the NE and SW images (separated by $1^{\prime \prime}$ ), would provide an interesting independent $T_{\mathrm{CMB}}$ measurement.

Additional transitions/molecules could be observed (within a short time interval to minimize time variations) in order to place tighter constraints on the physical conditions. However, and very importantly, collisional rates (with $\mathrm{H}_{2}$ ) are still unknown for a large number of molecules. This work illustrates the importance of obtaining these rates (see e.g. van der Tak 2011, for a short review on the field).

We have shown that the use of molecular transitions as diagnostics of physical conditions toward molecular absorbers is a powerful method with which to measure $T_{\mathrm{CMB}}$. Unfortunately, there is only a small number of $z>0$ millimeter-wave band molecular absorbers known to date (see a review by Combes 2008; and also Curran et al. 2011). Due to the brightness of the background continuum, the large column density of absorbing gas, the large number of detected molecules and the multiple independent lines of sight, the absorber toward PKS 1830-211 is a prime target for a precise measurement of $T_{\mathrm{CMB}}$. Another relatively well-suited target could be the $z=0.68$ absorber located toward the quasar B $0218+357$ (Wiklind \& Combes 1995). The physical conditions have already been explored using transitions of ammonia (Henkel et al. 2005) and formaldehyde (Jethava et al. 2007; Zeiger \& Darling 2010), but a thorough investigation combining several molecules, remains to be done for this source. However, a potentially severe difficulty is the small angular separation of $\sim 0.3^{\prime \prime}$ between the two gravitationally lensed images of the quasar.
In the (near) future, new $z>0$ molecular absorbers should be discovered (e.g. with the Atacama Large Millimetersubmillimeter Array or the Karl G. Jansky Very Large Array), providing many more opportunities to test the $T_{\mathrm{CMB}}$ evolution using molecular excitation analysis.

\section{Summary and conclusions}

We have performed a multi-transition excitation analysis of various molecular absorption lines toward the radio-mm molecular absorber PKS 1830-211, with the aim to precisely determine the CMB temperature at $z=0.89$. We use new 7 and $3 \mathrm{~mm}$ ATCA observations obtained in 2011, complemented with 2009 data from our ATCA $7 \mathrm{~mm}$ survey (Muller et al. 2011). Our results can be summarized as follows:

- The rotation temperatures of the observed molecules are all close to $5 \mathrm{~K}$, comparable to the value $T_{\mathrm{CMB}}=5.14 \mathrm{~K}$ predicted from the adiabatic expansion of the Universe. This suggests that the molecular excitation is mostly radiatively coupled with the $\mathrm{CMB}$, and that an accurate value of $T_{\mathrm{CMB}}$ can be derived.

- We combine a Monte-Carlo Markov chain approach with non-LTE radiative transfer predictions from the RADEX code to solve for the physical conditions of the absorbing gas, taking into account time variations of the quasar (i.e. possible changes of the sightline) between the 2009 and 2011 observations. For each epoch, we assume that the absorbing screen is homogeneous, and characterized by a single kinetic temperature and $\mathrm{H}_{2}$ density. Under this (reasonable) assumption, and with the molecular data available, we determine a CMB temperature of $5.08 \pm 0.10 \mathrm{~K}(68 \%$ confidence level) at $z=0.89$, which is the most precise measurement of $T_{\mathrm{CMB}}$ at $z>0$ to date.

- Combining this result with other measurements published in the literature, we refine the evolution of the CMB temperature as a function of redshift, $T=T_{0} \times(1+z)^{(1-\alpha)}$, finding $\alpha=0.009 \pm 0.019$, i.e. a quasi-linear dependence to the redshift. As far as we can tell, this is consistent with adiabatic expansion of the Universe.

- The absorbing gas at $z=0.89$ has properties similar to Galactic diffuse/translucent clouds, with a kinetic temperature of $\sim 80 \mathrm{~K}$ and a $\mathrm{H}_{2}$ density on the order of $10^{3} \mathrm{~cm}^{-3}$.

- We report the detection of several new species toward PKS 1830-211: $\mathrm{NH}_{2} \mathrm{CHO},{ }^{30} \mathrm{SiO}, \mathrm{HCS}^{+}$and tentatively $\mathrm{HOCO}^{+}$. The weak lines of these species could be detected in 2011 data toward the SW component owing to the general increase in absorption depth of all species by a factor of two compared to our previous observations in 2009.

This work emphasizes the importance of accurate molecular collisional data for astrophysical studies.

Acknowledgements. We thank the anonymous referee and the Editor, Malcolm Walmsley, for useful comments and suggestions to improve the presentation of the paper. This work makes intensive use of the LAMDA Database and RADEX program, we thank all the people who contributed to these projects. We thank A. Faure for providing us with an extended molecular data file for $\mathrm{HC}_{3}$ N. A.B. would like to thank M. Douspis for fruitful discussions and endless candies. The Centre for All-Sky Astrophysics is an Australian Research Council Centre of Excellence, funded by grant CE110001020. The Australia Telescope Compact Array is part of the Australia Telescope which is funded by the Commonwealth of Australia for operation as a National Facility managed by CSIRO. Based on observations carried out with the IRAM Plateau de Bure Interferometer. IRAM is supported by INSU/CNRS (France), MPG (Germany) and IGN (Spain). 


\section{Appendix A: spectra and fit results}

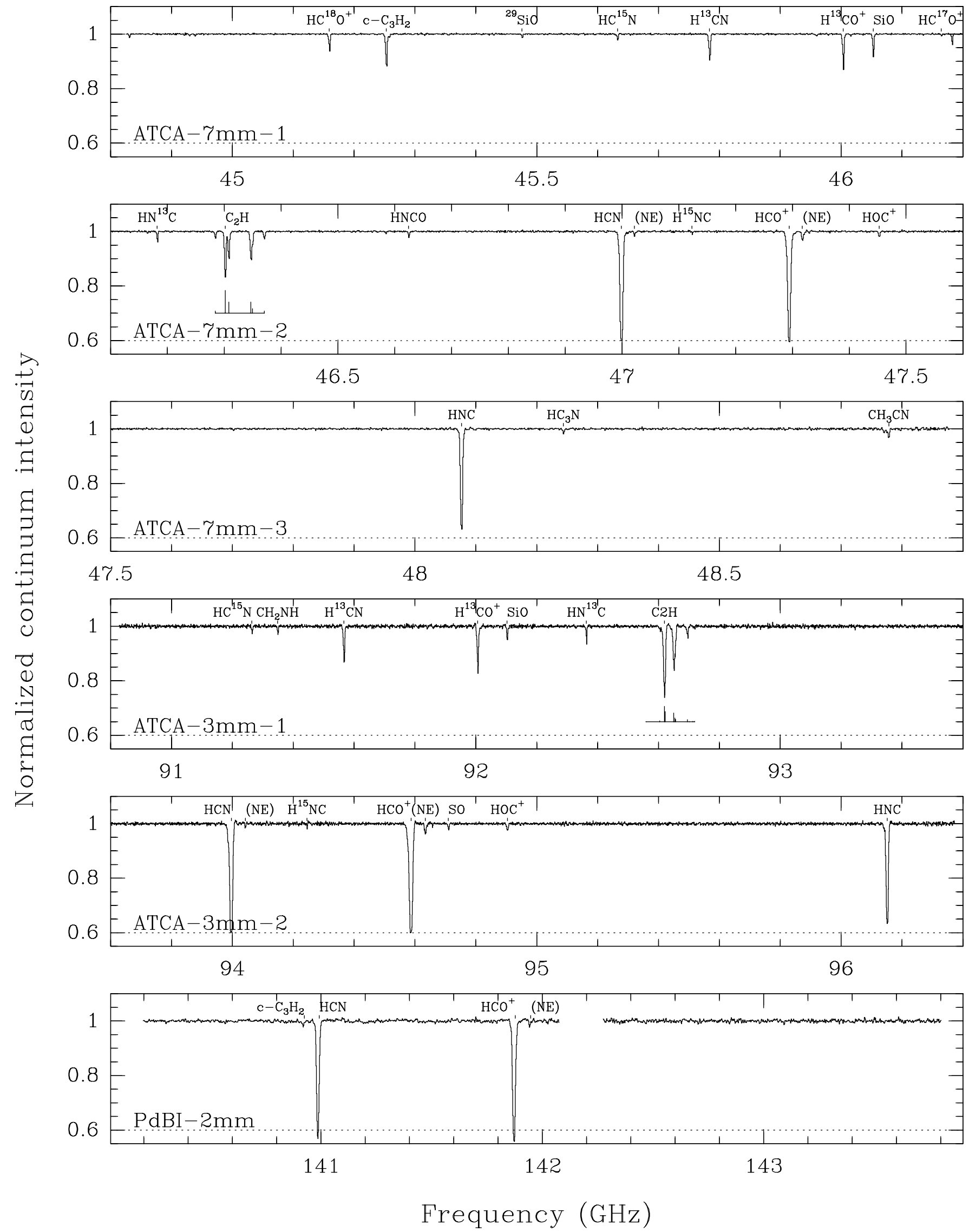

Fig. A.1. Spectra from ATCA and PdBI observations. The frequency scale is in the observer frame. The spectra are normalized to the total flux density corresponding to the sum of the NE and SW images. 


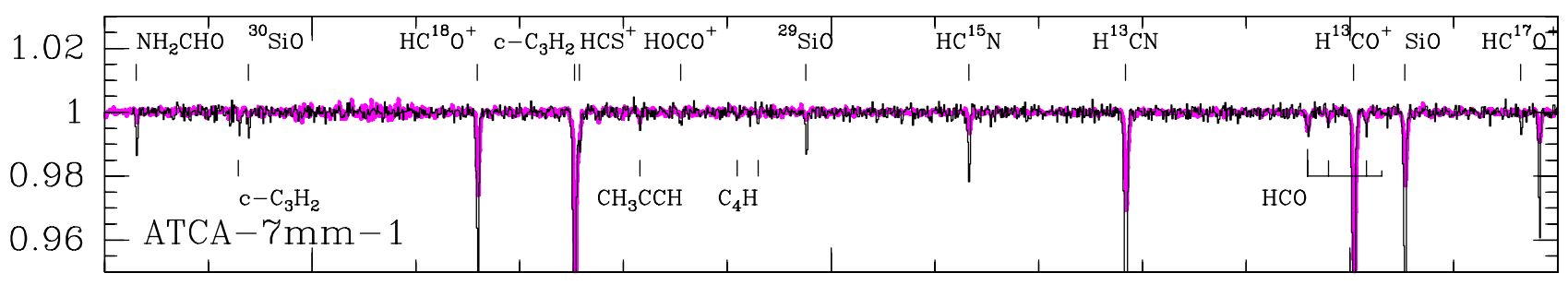
45

45.5

46

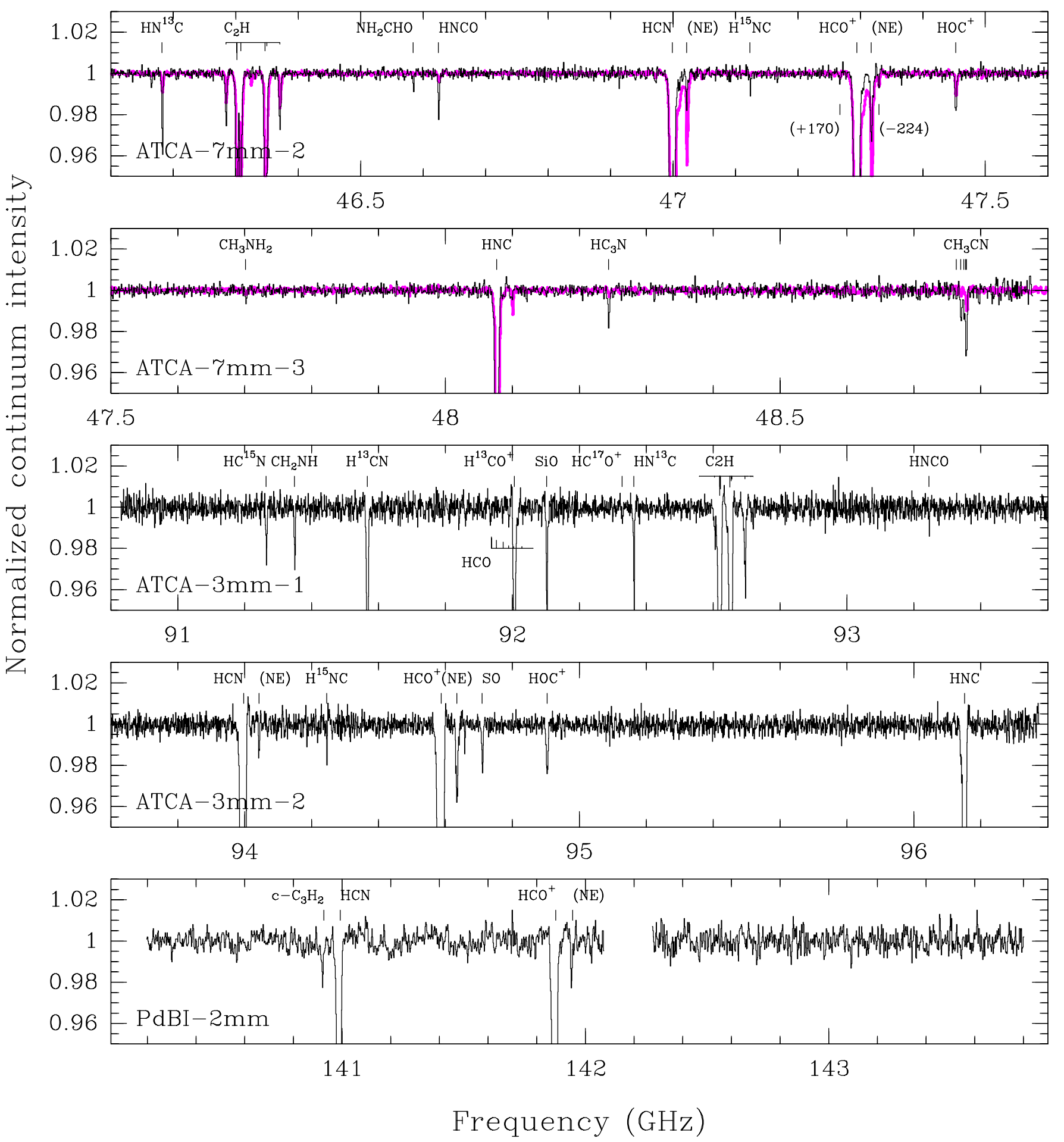

Fig. A.2. As Fig. A.1, but zoomed-in to emphasize the weak lines and with 2009/2010 averaged 7 mm spectrum overlaid (magenta). 
Table A.1. Parameters and results of the global fit of the spectra for non-saturated lines detected toward the SW component in 2011 ATCA observations.

\begin{tabular}{|c|c|c|c|c|c|c|}
\hline $\begin{array}{l}\text { Rest Freq. } \\
(\mathrm{MHz})\end{array}$ & Transition & $S_{u l}^{a}$ & $\begin{array}{c}E_{l} / k_{\mathrm{B}}^{b} \\
(\mathrm{~K})\end{array}$ & $\begin{array}{c}V_{0}{ }^{c} \\
\left(\mathrm{~km} \mathrm{~s}^{-1}\right)\end{array}$ & $\begin{array}{c}\Delta V^{d} \\
\left(\mathrm{~km} \mathrm{~s}^{-1}\right)\end{array}$ & $\begin{array}{c}\int \tau \mathrm{d} V^{e} \\
\left(10^{-3} \mathrm{~km} \mathrm{~s}^{-1}\right)\end{array}$ \\
\hline 87316.898 & $\mathrm{C}_{2} \mathrm{H} \mathrm{N}=1-0$ & 1.00 & 0.0 & $-0.5(0.1)$ & $18.9(0.1)$ & 28234 (280) \\
\hline 174663.199 & $\mathrm{C}_{2} \mathrm{H} N=2-1$ & 2.00 & 4.2 & $-{ }^{t}$ & $-{ }^{t}$ & $37574(486)$ \\
\hline 86339.922 & $\mathrm{H}^{13} \mathrm{CN} J=1-0$ & 1.00 & 0.0 & $-2.7(0.1)$ & $16.8(0.2)$ & $4966(69)$ \\
\hline 172677.851 & $\mathrm{H}^{13} \mathrm{CN} J=2-1$ & 2.00 & 4.1 & $-{ }^{t}$ & $-{ }^{t}$ & 6393(141) \\
\hline 86054.966 & $\mathrm{HC}^{15} \mathrm{~N} J=1-0$ & 1.00 & 0.0 & $-{ }^{t}$ & $-{ }^{t}$ & $945(42)$ \\
\hline 172107.957 & $\mathrm{HC}^{15} \mathrm{~N} J=2-1$ & 2.00 & 4.1 & $-{ }^{t}$ & $-{ }^{t}$ & 943(99) \\
\hline 90663.568 & HNC $J=1-0$ & 1.00 & 0.0 & $0.17(0.03)$ & $19.8(0.1)$ & 43339 (1130) \\
\hline 181324.758 & HNC $J=2-1$ & 2.00 & 4.4 & $-{ }^{t}$ & $-{ }^{t}$ & 45856 (1347) \\
\hline 87090.825 & $\mathrm{HN}^{13} \mathrm{C} J=1-0$ & 1.00 & 0.0 & $-t$ & $-^{t}$ & $1515(50)$ \\
\hline 174179.411 & $\mathrm{HN}^{13} \mathrm{C} J=2-1$ & 2.00 & 4.2 & $-{ }^{t}$ & $-{ }^{t}$ & $1778(86)$ \\
\hline 88865.715 & $\mathrm{H}^{15} \mathrm{NC} J=1-0$ & 1.00 & 0.0 & $-{ }^{t}$ & $-^{t}$ & $300(50)$ \\
\hline 86754.288 & $\mathrm{H}^{13} \mathrm{CO}^{+} J=1-0$ & 1.00 & 0.0 & $-2.7(0.1)$ & $15.6(0.1)$ & $6089(79)$ \\
\hline 173506.700 & $\mathrm{H}^{13} \mathrm{CO}^{+} J=2-1$ & 2.00 & 4.2 & $-t^{t}$ & $-{ }^{t}$ & 8072 (153) \\
\hline 85162.223 & $\mathrm{HC}^{18} \mathrm{O}^{+} J=1-0$ & 1.00 & 0.0 & $-{ }^{t}$ & $-{ }^{t}$ & $2581(46)$ \\
\hline 87057.535 & $\mathrm{HC}^{17} \mathrm{O}^{+} J=1-0$ & 1.00 & 0.0 & $-^{t}$ & $-^{t}$ & $262(40)$ \\
\hline 174113.169 & $\mathrm{HC}^{17} \mathrm{O}^{+} J=2-1$ & 2.00 & 4.2 & $-{ }^{t}$ & $-{ }^{t}$ & $231(70)$ \\
\hline 89487.414 & $\mathrm{HOC}^{+} J=1-0$ & 1.00 & 0.0 & $-0.2(0.5)$ & $19.3(1.1)$ & $1057(62)$ \\
\hline 178972.051 & $\mathrm{HOC}^{+} J=2-1$ & 2.00 & 4.3 & $-t^{t}$ & $-t^{t}$ & $1283(92)$ \\
\hline 86670.760 & $\mathrm{HCO} \mathrm{N} \mathrm{N}_{K}=1_{01}-0_{00} J=3 / 2-1 / 2$ & 1.00 & 0.0 & $-1.7(1.0)$ & $18.8(2.4)$ & $833(62)$ \\
\hline 172266.853 & $\mathrm{CH}_{2} \mathrm{NH} 2_{11}-2_{02}$ & 7.40 & 9.2 & $-4.1(0.6)$ & $9.8(1.4)$ & $812(61)$ \\
\hline 89956.068 & $\mathrm{CH}_{3} \mathrm{NH}_{2}(p) 1_{10}-1_{01}$ & 6.00 & 2.1 & $-4.8(1.5)$ & $12.3(3.2)$ & $223(53)$ \\
\hline 85338.893 & $\mathrm{c}-\mathrm{C}_{3} \mathrm{H}_{2}-\mathrm{o} 2{ }_{12}-1_{01}$ & 4.50 & 2.3 & $-1.9(0.1)$ & $17.8(0.2)$ & $6973(85)$ \\
\hline 84727.696 & $\mathrm{c}-\mathrm{C}_{3} \mathrm{H}_{2}(\mathrm{p}) 3_{22}-3_{13}$ & 0.96 & 12.1 & $-{ }^{t}$ & $-{ }^{t}$ & $244(38)$ \\
\hline 91958.726 & $\mathrm{CH}_{3} \mathrm{CN} J_{K}=5_{4}-4_{4}$ & 3.60 & 123.1 & $-3.9(0.5)$ & $14.6(1.1)$ & - \\
\hline 91971.130 & $\mathrm{CH}_{3} \mathrm{CN} J_{K}=5_{3}-4_{3}$ & 12.80 & 73.1 & $-{ }^{t}$ & $-{ }^{t}$ & - \\
\hline 91979.994 & $\mathrm{CH}_{3} \mathrm{CN} J_{K}=5_{2}-4_{2}$ & 8.40 & 37.4 & $-{ }^{t}$ & $-{ }^{t}$ & - \\
\hline 91985.314 & $\mathrm{CH}_{3} \mathrm{CN} J_{K}=5_{1}-4_{1}$ & 9.60 & 16.0 & $-{ }^{t}$ & $-^{t}$ & - \\
\hline 91987.088 & $\mathrm{CH}_{3} \mathrm{CN} J_{K}=5_{0}-4_{0}$ & 10.00 & 8.8 & $-{ }^{t}$ & $-{ }^{t}$ & - \\
\hline 87925.237 & $\mathrm{HNCO} 4_{04}-3_{03}$ & 4.00 & 6.3 & $-4.5(0.4)$ & $14.2(1.0)$ & $845(51)$ \\
\hline 175843.695 & HNCO $8_{08}-7_{07}$ & 8.00 & 29.5 & $-{ }^{t}$ & $-{ }^{t}$ & $257(81)$ \\
\hline 86846.960 & $\mathrm{SiO} J=2-1$ & 2.00 & 2.1 & $-3.0(0.1)$ & $14.4(0.2)$ & $3612(60)$ \\
\hline 173688.310 & $\mathrm{SiO} J=4-3$ & 4.00 & 12.5 & $-{ }^{t}$ & $-{ }^{t}$ & $1944(96)$ \\
\hline 85759.199 & ${ }^{29} \mathrm{SiO} J=2-1$ & 2.00 & 2.1 & $-{ }^{t}$ & $-^{t}$ & $541(37)$ \\
\hline 84746.170 & ${ }^{30} \mathrm{SiO} J=2-1$ & 2.00 & 2.0 & $-{ }^{t}$ & $-{ }^{t}$ & $287(35)$ \\
\hline 85347.890 & $\mathrm{HCS}^{+} J=2-1$ & 2.00 & 2.0 & $-1.5(0.0)^{f}$ & $15.0(1.4)$ & $542(45)$ \\
\hline 85531.512 & $\mathrm{HOCO}^{+} 4_{04}-3_{03}$ & 4.00 & 6.2 & $0.5(2.3)$ & $17.8(5.5)$ & $181(48)$ \\
\hline 84542.329 & $\mathrm{NH}_{2} \mathrm{CHO} 4_{04}-3_{03}$ & 12.00 & 6.1 & $-4.4(0.8)$ & $15.0(0.0)^{f}$ & $507(52)$ \\
\hline 87848.873 & $\mathrm{NH}_{2} \mathrm{CHO} 4_{13}-3_{12}$ & 11.23 & 9.3 & $-t^{t}$ & $-t$ & $290(42)$ \\
\hline 178605.403 & $\mathrm{SO} J_{K}=5_{4}-4_{3}$ & 4.91 & 15.9 & $-3.4(0.7)$ & $13.7(1.6)$ & $843(85)$ \\
\hline 90979.023 & $\mathrm{HC}_{3} \mathrm{~N} J=10-9$ & 10.00 & 19.6 & $-2.5(0.7)$ & $14.6(1.6)$ & $680(66)$ \\
\hline
\end{tabular}

Notes. ${ }^{(a)}$ Line strength. ${ }^{(b)}$ Energy of the lower level of the transition. ${ }^{(c)}$ Line centroid. ${ }^{(d)}$ FWHM linewidth. ${ }^{(e)}$ Integrated opacity. ${ }^{(t)}$ The parameter was tied with the precedent entry. ${ }^{(f)}$ The value was fixed. For some species, the fit results might be slightly different (although within uncertainties) to the values given in Table 3 , due to the different fitting procedure with tied parameters.

\section{References}

Battistelli, E. S., De Petris, M., Lamagna, L., et al. 2002, ApJ, 580, 101 Combes, F. 2008, Ap\&SS, 313, 321

Combes, F., \& Wiklind, T. 1999, in Highly Redshifted Radio Lines, eds. C. L. Carilli, S. J. E. Radford, K. M. Menten, \& G. L. Langston, ASP Conf. Ser., 156,210

Curran, S. J., Whiting, M. T., Combes, F., et al. 2011, MNRAS, 416, 2143 de Martino, I., Atrio-Barandela, F., da Silva, A., et al. 2012, ApJ, 757, 144

Dunkley, J., Bucher, M., Ferreira, P. G., Moodley, K., \& Skordis, C. 2005, MNRAS, 356, 925

Fixsen, D. J. 2009, ApJ, 707, 916

Garrett, M. A., Nair, S., Porcas, R. W., \& Patnaik, A. R. 1997, Vistas Astron., 41,281

Ge, J., Bechtold, J., \& Black, J. H. 1997, ApJ, 474, 67
Henkel, C., Jethava, N., Kraus, A., et al. 2005, A\&A, 440, 893

Henkel, C., Braatz, J. A., Menten, K. M., \& Ott, J. 2008, A\&A, 485, 451 Henkel, C., Menten, K. M., Murphy, M. T., et al. 2009, A\&A, 500, 725 Horellou, C., Nord, M., Johansson, D., \& Lévy, A. 2005, A\&A, 441, 435 Jauncey, D. L., Reynolds, J. E., Tzioumis, A. K., et al. 1991, Nature, 352, 132 Jethava, N., Henkel, C., Menten, K. M., Carilli, C. L., \& Reid, M. J. 2007, A\&A, 472,435

Jetzer, P., \& Tortora, C. 2011, Phys. Rev. D, 84, 043517

Jin, C., Garrett, M. A., Nair, S., et al. 2003, MNRAS, 340, 1309 Lidman, C., Courbin, F., Meylan, G., et al. 1999, ApJ, 514, 57

Lima, J. A. S. 1996, Phys. Rev. D, 54, 2571

Lima, J. A. S., Silva, A. I., \& Viegas, S. M. 2000, MNRAS, 312, 747 Liszt, H. S. 2012, A\&A, 538, A27

Lovell, J. E. J., Reynolds, J. E., Jauncey, D. L., et al. 1996, ApJ, 472, 5 Lovell, J. E. J., Jauncey, D. L., Reynolds, J. E., et al. 1998, ApJ, 508, L51 
Lu, L., Sargent, W. L. W., Barlow, T. A., et al. 1996, ApJS, 107, 475

Luzzi, G., Shimon, M., Lamagna, L., et al. 2009, ApJ, 705, 1122

Menten, K. M., Carilli, C. L., \& Reid, M. J. 1999, ASP Conf. Ser., 156, 218

Meyer, D. M., Black, J. H., Chaffee, F. H. Jr., Foltz, C. B., \& York, D. G. 1986, ApJ, 308, L37

Molaro, P., Levshakov, S. A., Dessauges-Zavadsky, M., \& D’Odorico, S. 2002, A\&A, 381, 64

Muller, S. 2011, IAU Symp., 280, 339

Muller, S., \& Guélin, M. 2008, A\&A, 491, 739

Muller, S., Guélin, M., Dumke, M., et al. 2006, A\&A, 458, 417

Muller, S., Beelen, A., Guélin, M., et al. 2011, A\&A, 535, A103

Nair, S., Jin, C., \& Garrett, M. A. 2005, MNRAS, 362, 1157

Noterdaeme, P., Petitjean, P., Srianand, R., et al. 2011, A\&A, 526, L7

Roth, K. C., \& Bauer, J. M. 1999, ApJ, 515, 57

Sault, R. J., Teuben, P. J., \& Wright, M. C. H. 1995, in Astronomical Data Analysis Software and Systems IV, eds. R. Shaw, H. E. Payne, \& J. J. E. Hayes, ASP Conf. Ser., 77, 433

Schöier, F. L., van der Tak, F. F. S., van Dishoeck, E. F., \& Black, J. H. 2005, A\&A, 432,369
Snow, T. P., \& McCall, B. J. 2006, ARA\&A, 44, 367

Songaila, A., Cowie, L. L., Hogan, C. J., \& Rugers, M. 1994a, Nature, 368, 599 Songaila, A., Cowie, L. L., Vogt, S., et al. 1994b, Nature, 371, 43

Srianand, R., Petitjean, P., \& Ledoux, C. 2000, Nature, 408, 931

van der Tak, F. 2011, IAU Symp., 280, 449

van der Tak, F. F. S., Black, J. H., Schöier, F. L., Jansen, D. J., \& van Dishoeck, E. F. 2007 , A\&A, 468, 627

Wernli, M., Wiesenfeld, L., Faure, A., \& Valiron, P. 2007a, A\&A, 464, 1147

Wernli, M., Wiesenfeld, L., Faure, A., \& Valiron, P. 2007b, A\&A, 475, 391

Wiklind, T., \& Combes, F. 1995, A\&A, 299, 382

Wiklind, T., \& Combes, F. 1996, Nature, 379, 139

Wiklind, T., \& Combes, F. 1998, ApJ, 500, 129

Wiklind, T., \& Combes, F. 1999, Poster contribution to Gravitational Lensing:

Recent Progress and Future Goals, eds. T. G. Brainerd, \& C. S. Kochanek, Boston University, July 1999

Winn, J. N., Kochanek, C. S., McLeod, B. A., et al. 2002, ApJ, 575, 103

Wright, E. L., Mather, J. C., Bennett, C. L., et al. 1991, ApJ, 381200

Zeiger, B., \& Darling, J. 2010, ApJ, 709, 386 